\title{
Cuenta Anual del Presidente de la Sociedad de Neurología, Psiquiatría y Neurocirugía (SONEPSYN)
}

\author{
Presentada en la Asamblea General de Socios, duranteel 60 Congreso Chileno de N eurología, \\ Psiquiatría y Neurocirugía, Pucón, Noviembrede2005
}

El Directorio 2004-2005 de la Sociedad de Neurología, Psiquiatría y N eurocirugía (SONEPSYN) inició su gestión el 9 de Noviembre de 2004, luego de que fueran renovados todos los cargos, salvo el de presidente, durante el último congreso anual.

Como ha sido costumbre en los últimos años, me referiré a los aspectos generales de la gestión para luego detallar las actividades realizadas. En una exposición aparte, el Dr. Mario Rivera, nuestro Tesorero, entregará un informe del estado financiero de la sociedad.

\section{Aspectos Generales de la Gestión}

El Directorio mantuvo su estilo corporativo, no autocrático de gestión. Una vez más, el nivel de actividad desarrollado fue alto durante todo el año, característica compartida por los últimos Directorios desde que felizmente hace siete años se decidiera liberar a las autoridades principales de SONEPSYN de tener que organizar el congreso anual, medida que ha redundado en la realización de un número mayor, más diversificado y regular de actividades.

Asumiendo que esta cuenta puede no hacer justicia al esfuerzo de algunos socios cuya aporte al trabajo institucional merecería mayor y más detallado reconocimiento, intentaremos dar una visión panorámica, equilibrada, del quehacer corporativo. Empero, estimamos necesario destacar aspectos que sobresalen de la gestión reciente de este Directorio. En este sentido, mencionaré la atención prestada nuevamente a los aspectos éticos y relacionados con los necesarios vínculos que una sociedad como la nuestra debe mantener con los proveedores médicos, en especial con la industria farmacéutica. Enseguida, cabe celebrar el intenso trabajo desplegado en EMC, al cual nos referiremos en un momento más. Por último, el Directorio subraya la encomiable labor de auditoria contable, como de ordenamiento de nuestras finanzas llevada a cabo por el tesorero y el contador, como resultado de la cual hoy contamos con balances confiables y una situación tributaria absolutamente en regla.

\section{Detalle de las actividades realizadas}

\section{Línea Editorial}

Durante el año se verificaron tres lanzamientos de libros. En Julio se efectuó el lanzamiento de la segunda edición del libro "Psicopatología de la M ujer", ceremonia realizada en la Casa Central de la Universidad de Chile, que contó con la presencia del Rector de la Universidad de Chile y el Decano de M edicina de la misma universidad.

Ayer -en el contexto de este congreso- se llevó a cabo la presentación del "Tratado de Neurología Clínica", ansiado y mayúsculo proyecto que finalmente alcanzó su concreción.

Además, en septiembre realizamos el lanzamiento, en nuestro medio, del texto iberoamericano de psiquiatría "Psiquiatría, 2a edición", de los editores Renato Alarcón, Guido Mazzotti y Humberto Nicolini, publicado conjuntamente por Manual Moderno y la Organización Panamericana de la Salud. Pese a que, en rigor, no es un texto de la serie editorial de SONEPSYN, se estimó pertinente realizar su lanzamiento local, toda vez que se han hecho presentaciones del libro en otros países y además entre los autores se 
encuentran doce psiquiatras chilenos, todos socios de SONEPSYN. En su conjunto, la obra alcanza las 1.100 páginas, escritas por 162 autores, provenientes de 17 naciones.

\section{Revista Chilena de Neuro-Psiquiatría}

En Noviembre de 2004 se ratificó al Dr. Jorge Nogales-Gaete como editor por un período adicional (dos años) y en Febrero de 2005 se envió la solicitud de indexación de la revista al Index Medicus. Nuestra principal publicación periódica ha mantenido su regularidad y no sólo ha seguido al día en su versión impresa sino también en su versión electrónica. En efecto, el número tres de este año se encuentra en la página web corporativa.

\section{Folia Psiquiátrica}

El objetivo de recuperar la periodicidad de publicación durante 2005 no se ha cumplido todavía, a pesar de los esfuerzos realizados por conseguir el financiamiento total de la revista. Independiente de que están en curso las gestiones para lograr dicho propósito, lo anterior pone de re lieve la pertinencia de abrir un debate acerca de la sobreoferta de publicaciones en el área de la psiquiatría. El tema es motivo de la editorial de noviembre de nuestra página web.

\section{Boletín Informativo}

Ha seguido entregando las últimas versiones de las revistas más consultadas, así como resúmenes que los editores consideran de interés para la actualización profesional de nuestros socios. En Enero de 2005 asumió como Editor General y Editor de Psiquiatría el Dr. Jorge Sánchez. Como Editor de Neurología continuó el Dr. Roberto Maturana y en Marzo se sumó al equipo, como Editor de Neurocirugía, el Dr. Pablo Rodríguez. Si bien desde el punto de vista de la calidad hay un evidente progreso, por razones que no son de responsabilidad de los editores, sino dicen relación con asuntos técnicos, no salieron los números de agosto ni de septiembre del boletín y el de octubre salió con retraso. En la actualidad esta situación se ha regularizado. Cabe señalar que pese a que en neurología y neurocirugía los aportes han sido de muy buen nivel, ellos no han tenido la regularidad que habría sido deseable.

\section{Docencia y Formación Continua}

Con el objetivo de darle continuidad al trabajo del Sistema de Recertificación M édica Continua (SIREMEC), en Noviembre de 2004 el Directorio creó el cargo, por tres años, renovable, de Editor Adjunto de Educación Médica Continua (EMC), puesto que fue asumido por la Dra. Alejandra Armijo, quien, a la vez, es uno de los tres encargados de docencia de SONEPSYN. LOS otros dos son el Dr. Pedro Chaná, de neurología y el Dr. Rómulo Melo, de neurocirugía. Ambos representan a sus respectivas especialidades en SIREMEC.

En total SONEPSYN realizó ocho cursos y/o encuentros científicos de EM C, de los cuales cinco se efectuaron en regiones, Concepción (2), Antofagasta, Viña del Mar y Valdivia. A continuación se resumen las características principales de las actividades desarrolladas en psiquiatría:

Curso: Actualización en Trastornos Psiquiátricos Fecha: 1 y 2 de Abril de 2005

Directores: Dr. Benjamín Vicente

Dr. Pedro Rioseco

Lugar: Hotel El Araucano, Concepción

$\mathrm{N}^{\circ}$ de Asistentes: 29

Curso: Actualización en Trastornos del Estado del Ánimo

Fecha: 3 y 4 de Junio de 2005

Directores: Dr. Carlos M adariaga

Dr. Juan Carlos M éndez

Lugar: Hotel Diego de Almagro Costanera, Antofagasta

$\mathrm{N}^{\circ}$ Asistentes: 56

Curso: Actualización en Trastornos Psiquiátricos Fecha: 26 y 27 de Agosto de 2005

Directores: Dr. Tomás Baader

Dr. Claudio Espejo

Lugar: Universidad Austral, Valdivia

$\mathrm{N}{ }^{\circ}$ Asistentes: 38 
Curso: VI Encuentro Psicoterapéutico

Fecha: 4 al 7 de Agosto de 2005

Directores: Dr. Niels Biedermann

Dr. Guillermo de la Parra

Lugar: Conference Town - Reñaca

$\mathrm{N}^{\circ}$ Asistentes: 185

Curso: Actualización en Psiquiatría

Fecha: Julio a Diciembre de 2005

Lugar: Hotel Santiago Park Plaza

- Módulo I

Julio: 22 y 23 de Julio

Directores: Dr. Archibaldo Donoso

Dr. Fernando Ivanovic-Zuvic

Dr. Mario Seguel

Dra. Julia Santin

№ Asistentes: 59

- Módulo II

Fecha: 11 y 12 de Agosto

Directores: Dr. Pedro Retamal

Dr. Luis Risco

Dr. Jorge Cabrera

Dra. Dagmar Holmgren

Dr. Carlos Cruz

Dra. Lina Ortiz

№ Asistentes: 79

- M ódulo III

Fecha: 23 y 24 de Septiembre

Directores: Dr. César Carvajal

Dr. Alejandro Gómez

Dra. Patricia Rentería

Dr. Alejandro Koppmann

Dr. Gonzalo Poblete

№ Asistentes: 65

- Módulo IV

Fecha: 14 y 15 de 0 ctubre

Directores: Dr. Antonio M enchaca

Dr. Raúl Riquelme

Dr. Rogelio Isla

Dr. Daniel Martínez

Dr. Rodrigo Santis

№ Asistentes: 58
- Módulo V

Fecha: 11 y 12 de Noviembre de 2005

Directores: Dra. Rosa Behar

Dra. M ónica Sangüesa

Dra. Gloria Gramegna

Dr. Ricardo Capponi

Dr. Sergio Bernales

Dr. Luis Tapia

№ Asistentes: 41

- Módulo VI

Fecha: 14 y 15 de Diciembre

Directores: Dra. Verónica Larach

Dra. Sonia Tardito

Dr. Eduardo Durán

Dr. Eugenio Olea

Dr. Enrique Jadresic

Dr. Hernán Silva

En especial, merece destacarse el Curso de Actualización en Psiquiatría realizado en Santiago en módulos mensuales entre julio y diciembre de este año, pues se trata de un curso de gran extensión, que contempla la actualización de los grandes temas de la especialidad e incluye como docentes a nada menos que 85 especialistas de reconocida trayectoria.

En neurología se efectuaron los tradicionales dos cursos anuales, a saber:

\section{Curso:}

Actualización en N eurología 2005

"N ovedades Diagnósticas"

Fecha: 24 de Junio de 2005

Director: Dra. Violeta Díaz

Lugar: Centro de Eventos Club M anquehue

$\mathrm{N}^{\circ}$ de Asistentes: 121

\section{Curso:}

Actualización en N eurología 2005

"Novedades Terapéuticas"

Fecha: 23 de Septiembre de 2005

Director: Dr. Walter Feuerhake

Lugar: Centro de Eventos Club M anquehue № de Asistentes: 94 
En neurocirugía se llevó a cabo una actividad científica de carácter internacional, cuyas características se sintetizan a continuación:

Simposio Internacional Cirugía Base de Cráneo, VII Aniversario Grupo Chileno de Base de Cráneo Fecha: 9,10 y 11 de Junio de 2005

Directores: Dr. Jaime Pinto

Dr. Armando Ortiz

Dr. Tyler Frizzell

Lugar: Hotel El Araucano, Concepción

$\mathrm{N}^{\circ}$ Asistentes: 55

Además, hay otros dos logros que ameritan ser destacados. En primer lugar, el hecho de que, por primera vez, este año nuestra sociedad va a ser capaz de entregar el programa de actividades a realizar en un año determinado durante el año calendario anterior. En efecto, antes de concluido este año, SO NEPSYN va a publicar en la página web la programación para 2006. Desde ya les puedo anticipar que durante el próximo año realizaremos todas las actividades de EMC de Santiago en el mismo lugar. A su vez, podemos informar que efectuaremos, entre otros, un curso para no especialistas en el que integraremos nuestras tres especialidades. Enseguida, como ya se ha difundido, desde 2006 SONEPSYN ofrecerá periódicamente, a alguno de sus socios psiquiatras, la oportunidad de hacer una pasantía breve en la Clínica M ayo de los Estados U nidos. En paralelo, en neurología existirá un programa de financiamiento de pasajes, estadías o inscripciones a cursos, congresos o estadías de perfeccionamiento. Ambos programas serán financiados por SONEPSYN.

\section{Aspectos éticos}

Se ha recomendado a los expositores de nuestras actividades científicas, sean socios o no, que declaren sus potenciales conflictos de intereses antes de hacer sus presentaciones. Esta costumbre comenzó muy tímidamente hace dos año, consti- tuyéndose -como ustedes han podido apreciar durante este congreso- en una realidad creciente que el actual Directorio está tratando de convertir en parte de la cultura institucional de nuestros socios. Fue considerada en el seno del Directorio la posibilidad de hacer obligatoria dicha declaración pero, después de un amplio debate y de la consulta a diversas instancias, tales como anteriores presidentes de SONEPSYN, el Comité de Ética y socios destacados, se estimó preferible mantener por el momento el carácter voluntario de la declaración.

A su vez, durante el año se revisaron las pautas existentes sobre las relaciones de SONEPSYN con la industria que no se habían evaluado ni modificado desde su elaboración en 1999. Se actualizaron a la luz de los conocimientos que se han ido acumulando en torno al tema, nuevamente se consultó al Comité de Ética y otros expertos para, finalmente, en septiembre aprobarse una versión final, la cual el Directorio resolvió enviar para conocimiento de las instancias pertinentes.

\section{Página web}

Se realizó una encuesta a los socios para mejorar el servicio entregado. A la luz de sus resultados, durante el segundo semestre del año se modificó el diseño gráfico y se aumentó la frecuencia de actualización de la página. Además de los contenidos habituales, se han subido a la página importantes documentos, tales como la recomendación de SONEPSYN de hacer declaración de potenciales conflictos de intereses, documentos de ASOCIMED, de la Academia de Medicina y la versión en español de World Psychiatry, revista oficial de la Asociación Mundial de Psiquiatría, entre otros. La sección editorial ha mantenido su continuidad pero además se crearon tres nuevas secciones, "N ovedades", "Prensa" y "Galería de Fotos". En términos de visitas, ha habido un aumento ostensible en el número de ellas en comparación a hace un año atrás. Por ejemplo, en octubre el número de visitas diarias fluctuó entre 55 y 374, con una mediana sobre 120. 


\section{Grupos de Trabajo}

En cinco años se ha duplicado el número de Grupos de Trabajo (GDTs). Así, mientras en 2000 teníamos sólo 10 GDTs, hoy contamos con 21, dos de los cuales se crearon desde nuestra última cuenta anual, a saber: el GDT de Esquizofrenia -que tiene como coordinadora a la Dra. Alejandra Armijo-, aprobado como GDT "en formación" en Diciembre del año pasado y el GDT de Trastornos del Sueño (también "en formación") cuya aprobación se dictó en Mayo. La primera Coordinadora de este GDT fue la Dra. Julia Santín, quien en Julio fue reemplazada por el Dr. Alejandro De Marinis.

Cabe consignar que en enero se actualizó el reglamento de los GDTs, siendo aprobada la nueva versión, y subida a la página web, en Marzo de 2005.

\section{Comisiones}

Aparte de las comisiones de especialidad, las comisiones Gremial, de D ocencia y Ética han continuado trabajando en forma activa. Las contribuciones a los temas éticos y de docencia ya se han mencionado. A la Comisión Gremial se incorporó el Dr. Reynaldo Bustos, de diligente y reconocida trayectoria en el Colegio M édico de Chile. Nuestra sociedad ha seguido teniendo representantes ante el Ministerio de Salud y colaborando, tanto en neurología como en psiquiatría, con el plan AUGE y la Comisión de Clasificación Internacional de Funcionalidad del M IN SAL. Como iniciativa inédita, se debe destacar que este año por primera vez nuestra sociedad se presenta a un concurso para fondos de investigación; específicamente, se trata de una postulación al FONIS, con el objeto de llevar a cabo un estudio epidemiológico sobre enfermedades neurológicas. También en el ámbito neurológico, es elogiable la formación de dos comisiones abocadas a definir los criterios para la certificación de la práctica en electromiografía y electroencefalografía, las cuales deberían estar concluyendo su trabajo en diciembre de este año, como asimismo la realización, durante este congreso anual, del Primer Claustro de la especialidad.

\section{Vínculos Nacionales}

Junto a las actividades de colaboración con el $M$ inisterio de Salud y el Colegio M édico, este año se mantuvo nuestra participación en CON ACEM y ASOCIMED. Las recomendaciones de esta última asociación, referidas al control del conflicto de intereses, fueron difundidas a nuestros socios. A su vez, respondimos a la convocatoria que hizo ASOCIMED para entregar el Premio Nacional de M edicina 2006, postulando a dicha distinción a nuestro distinguido Maestro de la Neurología Dr. Fernando Novoa. Esta postulación fue en conjunto con la Sociedad de Psiquiatría y Neurología de la Infancia y la Adolescencia (SOPNIA).

\section{Relaciones Internacionales}

SONEPSYN continúa representando a nuestras especialidades en distintos organismos internacionales. La sociedad no sólo tuvo una significativa presencia en el XIII Congreso Mundial de Psiquiatría, efectuado en El Cairo, Egipto, evento al que fue becada por la Asociación M undial de Psiquiatría (WPA) nuestra socia Dra. Katherina Llanos, sino también fue comisionada por la Asociación M undial de Psiquiatría para representar a la asociación mundial de especialistas en la Asamblea M édica M undial, realizada en Santiago desde el 12 al 15 de Octubre.

En neurología, cabe mencionar la integración a la Federación de Sociedades Iberoamericanas de Neurología y la participación de socios nuestros en los programas de EM C para el año 2006, de la Federación Mundial de Neurología. Adicionalmente, quisiera destacar el sobresaliente desempeño de la neurología nacional, representada por los doctores Renato Verdugo y Francisco Soto en el Tournament of the mind, realizado recientemente en el Congreso Mundial de Neurología. Pese a ser sólo una dupla, en esta competencia académica obtuvieron el séptimo lugar entre treinta países participantes, resultando -holgadamente- los mejores de Hispanoamérica, superando, entre otros, a España y Estados U nidos. 


\section{Aspectos administrativos}

Se aplicó el sistema de calificación cuatrimestral del personal, introducido el año 2004. A contar del 3/5 se desvinculó de nuestra a institución a la Srta. Evelyn Sepúlveda, siendo asumidas temporalmente sus funciones por la Sra. Susana Salvadores y la Sra. Victoria M erello. A partir de Julio asumió como Secretaria Editorial la Sra. Ana Cruces. Además, se desempeñaron durante un tiempo en SONEPSYN dos periodistas. Desde abril a agosto lo hizo la Sra. Verónica González y desde Septiembre a N oviembre la Sra. Elizabeth Simonsen. En lo que atañe a los bienes, se sustituyó la antigua fotocopiadora por una nueva y se vendió la motocicleta que durante un tiempo usó el estafeta que tuvimos.

\section{Nuevos socios}

Desde nuestra última cuenta anual ingresaron 32 nuevos socios, 15 en neurología, 16 en psiquiatría y uno en neurocirugía.

NEUROLOGÍA:

Andrés Aragón G.

Evelyn Benavides $\mathrm{S}$.

Daniel Cárcamo P.

M aría Loreto Cid J.

María Duque $P$.

Jorge González H.

Mónica González S.

Arturo Jaramillo $M$.

Carolina Kunstmann R.

Gisela Küester F.

Mirta López G.

Verónica Olavarría I.

Luis Pedraza C.

Pedro Vasquez $\mathrm{S}$.

Raúl Valenzuela M.

PSIQUIATRÍA:

Rodrigo Aguirre D.

Luis Barra A.

Luis Botto V.

Jorge Calderón P.

María Patricia Cordella M.
Jorge Gaete 0.

$M$ iriam Haase $U$.

Daniel Martínez A.

Antonio Menchaca $P$.

Sofía Ortiz C.

Danilo Quiroz L.

Rául RiquelmeV.

Pablo Salinas T.

M ónica Sangüeza M .

M atías González T.

Gonzalo Poblete A.

NEUROCIRUGÍA

Patricio Bustos $\mathrm{G}$.

\section{Actividades culturales}

Por cuarto año consecutivo, SONEPSYN organizó su tradicional concurso de pintura, cuya convocatoria se centró esta vez en "La enfermedad en el Arte". Las obras se encuentran en exhibición en las dependencias del congreso. Durante el año se publicó el catálogo de la versión anterior de este concurso, el cual incluye un prólogo del artista visual Gonzalo Cienfuegos. Para el próximo año, se encuentra totalmente organizado un taller de Psiquiatría y Cine.

\section{Extensión}

Hemos incorporado esta sección a nuestro informe para dar cuenta de un trabajo de acercamiento a la comunidad en el que está empeñado nuestra sociedad. En la línea de colaborar a la educación médica de la población y entregar la opinión autorizada de las especialidades que congrega SONEPSYN, se han llevado a cabo varias iniciativas. La principal de ellas fue la organizada por el Dr. Pedro Retamal, denominada "Semana de la Depresión", en el contexto de la cual se realizaron diversas actividades. Entre ellas, una conferencia de prensa, desarrollada en la Facultad de M edicina de la U niversidad de Chile y una M esa Redonda, que tuvo lugar en la Clínica Santa María, en las que estuvieron presentes, entre otros, el decano de Medicina y el ministro de salud, respectivamente. La semana incluyó la dis- 
tribución de 50.000 dípticos educativos, como también la habilitación de 13 casillas de internet para que el público pudiera, durante tres días, hacer preguntas en forma gratuita, las cuales fueron contestadas por psiquiatras jóvenes voluntarios. Se respondió un total de 116 correos electrónicos. Además, durante el año nuestra sociedad se pronunció, a través de cartas al director de medios de prensa y artículos de opinión, sobre temas que fueron de interés público, entre ellos el debate en torno a la nueva ley de control del tabaco y los problemas de salud mental asociados al crecimiento económico y la desigualdad social.

\section{Congreso Anual 2006}

En Abril se nombró al Dr. Luis Risco como Director del Comité Organizador del 61 Congreso Anual de SONEPSYN, cuyo equipo se encuentra trabajando activamente. Dicho congreso se realizará en la ciudad de La Serena, desde el 2 al 4 de Noviembre de 2006. A este respecto, corresponde informar que recientemente se aprobó un Reglamento de Organización de Congresos
SONEPSYN, el que fue elaborado por miembros del Directorio que han participado en la organización de congresos anteriores, el que esperamos sea de utilidad para los futuros encargados de nuestra principal actividad académica anual.

Deseo concluir esta cuenta con un sincero reconocimiento al Directorio y a cada uno de los socios que, al igual que otros en el pasado, durante este período han contribuido - con su aporte a hacer de SONEPSYN lo que es, una prestigiosa sociedad científica, y un grato espacio para el encuentro y la convivencia. Además, quiero felicitar a los gestores de este 60 Congreso anual, doctores Rómulo M elo, David Rojas, Roberto Maturana, Jaime Santander, Manuel M orales y Pablo Arancibia, por entregarnos un magnífico congreso. Por último, en nombre del Directorio hago llegar nuestro agradecimiento al equipo de secretarias, en especial a Susana Salvadores, y al contador, Sr. Marcos Contreras, por su inestimable apoyo.

\section{Dr. Enrique Jadresic M. Presidente SONEPSYN}




\section{Índice de Revisores-Árbitros 2005}

\begin{tabular}{ll} 
A & L \\
Aboitiz, Francisco & Lavados, Pablo \\
C & Lolas, Fernando \\
Carvajal, César & M \\
Cabrera, Jorge & Matus, Cristian \\
CH & N \\
Chaná, Pedro & Nader, Armando \\
D & Nogales-Gaete, Jorge \\
De la Barra, Flora & \\
Díaz, Fernando & R \\
Donoso, Archibaldo & Rees, Reginald \\
& Rioseco S. Pedro \\
F & Rivera, Mario \\
Figueroa, Gustavo & \\
Fuentes, Patricio & S \\
G & Saez, David \\
Godoy, Jaime & Salinas, Rodrigo \\
González H. Jorge & Sánchez, Jorge \\
H & Silva, Hernán \\
Heerlein, Andrés & Slachevsky, Andrea \\
I & Servat, Mónica \\
Ivanovic-Zuvic, Fernando & \\
\hline J & T \\
Juri, Carlos & Tagle, Patricio \\
\hline & V \\
\hline & Verdugo, Renato \\
& Von Bernhardi, Rommy \\
\end{tabular}

